\title{
Metabolic syndrome is associated with improved cancer-specific survival in patients with localized clear cell renal cell carcinoma
}

\author{
Zhenhua Liu" ${ }^{1 \#}$, Haifeng Wang ${ }^{1 \#, *}$, Lian Zhang ${ }^{1 \&}$, Shaobo Li $^{2}$, Yu Fan ${ }^{1}$, Yisen Meng ${ }^{1}$, Shuai Hu ${ }^{1}$, Qian Zhang ${ }^{1}$, \\ Zhisong $\mathrm{He}^{1}$, Liqun Zhou ${ }^{1}$, Wenke Han ${ }^{1}$, Wei Yu ${ }^{1}$, Jie Jin ${ }^{1}$ \\ ${ }^{1}$ Department of Urology, Peking University First Hospital and Institute of Urology, National Research Center for Genitourinary Oncology, Beijing \\ 100034, China; ${ }^{2}$ School of Basic Medicine, Fudan University, Shanghai 200032, China \\ Contributions: (I) Conception and design: W Yu, J Jin; (II) Administrative support: Q Zhang, Z He, L Zhou, W Han; (III) Provision of study materials \\ or patients: H Wang, Z Liu; (IV) Collection and assembly of data: L Zhang, S Hu; (V) Data analysis and interpretation: Y Fan, S Li, Y Meng; (VI) \\ Manuscript writing: All authors; (VII) Final approval of manuscript: All authors. \\ \#These authors contributed equally to this work as the co-first authors. \\ Correspondence to: Jie Jin, MD, PHD; Wei Yu, MD. Department of Urology, National Urological Cancer Center, Peking University First Hospital \\ and Institute of Urology, Peking University, 8 Xishiku Street, Xicheng District, Beijing 100034, China. Email: jinjie@vip.163.com; yuweif@126.com.
}

\begin{abstract}
Background: Metabolic syndrome (MetS) has been found to be prevalent in cancer and have implications in cancer outcomes. In this study, we attempted to evaluate the prognostic value of MetS in localized clear cell renal cell carcinoma (ccRCC) patients.

Methods: We retrospectively collected clinicopathological data and pre-treatment laboratory test results of 480 patients with localized (T1-2N0M0) ccRCC undergoing radical or partial nephrectomy in Peking University First Hospital. MetS was diagnosed by criteria of the 2004 Chinese Medical Association Diabetes Society. Univariate and multivariate analyses were conducted to analyze the association between clinicopathological characteristics, MetS, and disease outcomes.

Results: In our cohort, 136 patients (28.3\%) were diagnosed with MetS. Among them, 113 (83.1\%) were men, suggesting that men were more likely to have MetS. This syndrome was also associated with increased pre-treatment creatinine levels. Median follow-up time was 70 months (range, 1-118 months) and 5-year overall survival (OS) rate was $92 \%$. MetS was an independent favorable factor of cancer-specific survival (CSS) $(\mathrm{P}=0.017)$, and similar results were observed in Fuhrman nuclear grade 1-2 ccRCC patients by further analysis. Neither of the four components of the MetS (hypertension, diabetes mellitus, overweight/obesity and dyslipidemia) was an independent predictor of CSS. Patients who met more than 3 of the 4 criteria for MetS had higher CSS than those who met fewer than 2 criteria.
\end{abstract}

Conclusions: MetS is an independent prognostic factor for better CSS in localized ccRCC patients.

Keywords: Metabolic syndrome (MetS); renal cell carcinoma (RCC); prognosis; cancer-specific survival (CSS)

Submitted Jul 02, 2019. Accepted for publication Sep 29, 2019.

doi: $10.21037 /$ tau.2019.10.04

View this article at: http://dx.doi.org/10.21037/tau.2019.10.04

\footnotetext{
*, current address: Department of Anesthesia, Peking University First Hospital, 8 Xishiku Street, Xicheng District, Beijing 100034, China.

\& , current address: Department of Nephrology, Zhongnan Hospital of Wuhan University, Wuhan, China.
} 


\section{Introduction}

Renal cell carcinoma (RCC) is a common urologic tumor and accounts for about $5 \%$ in men and 3\% in women of all oncological diagnoses (1). There are more than 140,000 RCC-related deaths yearly, making RCC the 13 th most common cause of cancer death worldwide (1). However, the etiology of RCC remains largely unknown, despite the recognition of smoking and diet as risk factors. Recent decades have seen some improvement in its diagnosis and treatment, but RCC still remains an aggressive and often fatal disease (2). The 2016 WHO classification categorized RCC into several histological subtypes: clear cell, papillary, chromophobe, collecting duct, renal medullary, mucinous tubular and spindle, tubulosystic, clear cell papillary, unclassified RCC etc., (3). Of all the subtypes, the outcome of clear cell RCC (ccRCC) is unsatisfactory: about one-third of ccRCC patients experienced metastasis and up to one half treated for localized ccRCC experienced recurrence (4).

Metabolic syndrome (MetS), first described as "Syndrome X" by Reaven (5), is a group of metabolic abnormalities including central obesity, hypertension, hyperlipidemia, and hyperglycemia, with insulin resistance its pathological and primary denominator. Because of altered dietary pattern and lifestyle, MetS has been associated with increased morbidity in recent years. Approximately $20 \%$ of Chinese people had MetS (6), which has become a worldwide concern.

Increasing evidence shows that MetS is related to many cancers, with various effects on prognosis. MetS and its four components (hypertension, diabetes mellitus, overweight/obesity and dyslipidemia) were associated to a better outcome for gastric cancer (7) and tongue squamous cell cancer (8) but worse survival for breast cancer (9) and prostate cancer (10). RCC was increasingly being found to be a metabolic disease, with MetS prevalent in RCC patients (11). Previous studies concentrated on the effect of only one MetS factor on the prognosis of RCC, however, very few studies have evaluated the impact of MetS on survival with RCC.

Here we evaluated whether MetS plays a part on the prognosis of RCC.

\section{Methods}

\section{Patients}

We included 480 localized ccRCC (T1-2N0M0) patients with complete clinical data for screening MetS, in a single center, Peking University First Hospital from January
2005 to December 2010. All our subjects received surgical treatment (radical or partial nephrectomy). All the enrolled cases had negative surgical cut tumor edge. Pathological TNM stage for each RCC was determined according to the AJCC 2002 TNM staging system. Patients with unclear MetS status in our investigations were excluded. Patients were closely followed up after discharge with regular postoperative tests including blood and urine tests, radiography, urological ultrasonography, and CT when needed.

\section{Criteria used for the diagnosis of MetS}

We used criteria for MetS from the Chinese Medical Association Diabetes Society in 2004 (12). Patients with MetS had at least 3 of the following: (I) overweight and (or) obesity: body mass index (BMI) $\geq 25.0 \mathrm{~kg} / \mathrm{m}^{2}$; (II) fasting plasma glucose $\geq 6.1 \mathrm{mmol} / \mathrm{L}(110 \mathrm{mg} / \mathrm{dL})$ and/or 2-hr plasma glucose $\geq 7.8 \mathrm{mmol} / \mathrm{L}(140 \mathrm{mg} / \mathrm{dL})$ or drug treatment for diagnosed diabetes mellitus; (III) systolic or diastolic blood pressure $\geq 140 / 90 \mathrm{mmHg}$ or drug treatment for diagnosed hypertension; (IV) fasting serum triglycerides level $\geq 150 \mathrm{mg} / \mathrm{dL}(1.7 \mathrm{mmol} / \mathrm{L})$ and/or fasting serum highdensity lipoprotein (HDL) cholesterol level $<0.9 \mathrm{mmol} / \mathrm{L}$ $(35 \mathrm{mg} / \mathrm{dL})$ in men and $<1.0 \mathrm{mmol} / \mathrm{L}(39 \mathrm{mg} / \mathrm{dL})$ in women.

\section{Definitions of overall survival (OS), cancer-specific survival (CSS) and recurrence-free survival (RFS)}

OS, CSS, RFS were defined as the interval between the date of surgical treatment to the date of: (I) death or last follow-up; (II) cancer-related death or last follow-up; and (III) radiological or histological confirmation of cancer recurrence or last follow-up, respectively.

\section{Statistical analyses}

A Chi-square test was used to compare categorical clinicopathological parameters between patients with and without MetS. OS, CSS, RFS curves were plotted by the Kaplan-Meier method and differences by MetS states were weighed by $\log$-rank test. Significant variables in univariate analysis $(\mathrm{P} \leq 0.05)$ were included in the multivariate Cox proportional hazards regression model. Hazard ratios (HRs) and $95 \%$ confidence intervals (95\% CIs) were estimated. All statistical tests were two-sided and the significance level was set at $\mathrm{P}<0.05$. All analyses involved use of SPSS 22.0 for Windows. 


\section{Results}

\section{Characteristics of the cohort}

Among the 480 ccRCC patients, 364 were male (75.83\%); the mean age was 55.25 (range, 21-86 years). In total, 136 (28.3\%) and 344 (71.7\%) underwent partial nephrectomy or radical nephrectomy, respectively. Approximately $50 \%$ of RCC cases were on the left kidney; 47 patients $(9.79 \%)$ presented cancer-related symptoms [as the clinical presentation of RCC may include localized and systemic diseases (13)], RCC patients presented one or more of the following symptoms including flank pain, hematuria, fever and weight loss were considered with positive symptoms in this study) before treatment. A total of 418 (87.08\%) RCC patients were at pT1 and $62(12.92 \%)$ at pT2 stages. Overall, 136 patients (28.3\%) had MetS (Table 1).

\section{Association between MetS status and clinicopathological parameters}

Patients with and without MetS [136 (28.3\%) and 344 (71.7\%), respectively] did not differ significantly in age, tumor laterality, presence of symptoms, tumor size, pathological T stage (T1 and T2), histological type, Fuhrman nuclear grade, surgery method (open or laparoscopic, nephron sparing or radical nephrectomy) or tumor necrosis. MetS is more frequent in males than females $(\mathrm{P}=0.020)$. Higher pre-treatment creatinine level was more prevalent in MetS subjects $(\mathrm{P}<0.001)$. Nevertheless, both groups showed a mean creatinine level within the normal range.

\section{Association of MetS status and OS, CSS and RFS}

The median follow-up was 70 months (range, 1-118 months). Overall, 5 and 31 patients with and without MetS died, respectively. Median OS was 66 months (range, 1-118 months) and the 5 -year OS rate was $92 \%$. In univariate analysis, CSS was significantly longer for MetS patients $(\mathrm{P}=0.028)$; MetS patients also had a tendency to have better OS $(\mathrm{P}=0.093)$ than patients without MetS (Figure 1). In multivariate analysis, MetS remained an independent protective factor for ccRCC CSS (HR 0.168, 95\% CI: 0.039-0.728, $\mathrm{P}=0.017)$. MetS was not an independent factor for OS and RFS in this study.

\section{Other factors associated with OS, CSS, and RFS}

In univariate analysis (Table 2), larger tumor size and higher neutrophil-lymphocyte ratio (NLR) were associated with worse OS, CSS and RFS (all $\mathrm{P}<0.05$ ). Therefore, patients with smaller tumor sizes and lower NLR values may get a better prognosis. The presence of symptoms was related to worse CSS and RFS $(\mathrm{P}=0.047$ and $\mathrm{P}=0.002$, respectively), but not to OS $(\mathrm{P}=0.151)$. Elder age was correlated with worse OS and CSS $(\mathrm{P}=0.001$ and $\mathrm{P}=0.009$, respectively) but not with RFS $(\mathrm{P}=0.157)$. Higher Fuhrman grade status was statistically linked with worse OS and RFS $(\mathrm{P}=0.020$ and $\mathrm{P}<0.001$, respectively). What's more, higher Charlson comorbidity index (CCI) was related to worse OS and CSS ( $\mathrm{P}=0.005$ and $\mathrm{P}=0.024$, respectively).

In multivariate analysis (Table 3), the following factors were independent prognostic factors for both CSS and RFS: bigger tumor sizes indicate worse CSS (HR 4.166, 95\% CI: $1.632-10.1632, \mathrm{P}=0.003)$ and RFS (HR 3.702, 95\% CI: $1.905-7.194, \mathrm{P}<0.001)$; presence of symptoms was also a negative predictor for CSS (HR 2.841, 95\% CI: $1.040-7.762, \mathrm{P}=0.042)$ and RFS (HR 2.485, $95 \%$ CI: 1.246-4.956, P=0.010). Higher NLR $(\geq 3)$ was an independent risk factor for CSS (HR 3.096, 95\% CI: 1.394-6.876, $\mathrm{P}=0.005$ ).

\section{Effects of MetS on the prognosis of ccRCC with different Fubrman grades}

In different grading subgroups of ccRCC, will the predictive value of MetS still be preserved? To answer this question, we further divided our subjects into two subgroups based on their Fuhrman grades, and separately examined whether the presence of RCC was still related to cancer outcome. As shown in Table 4 and Figure 2, MetS status was associated with CSS only in RCC patients with Fuhrman nuclear grade $1-2$ by univariate analysis $(\mathrm{P}=0.031)$. Therefore, we conducted a multivariate analysis in Fuhrman grades 1-2 ccRCC patients and discovered MetS still remained an independent favorable prognostic factor for CSS (HR 0.103, 95\% CI: 0.014-0.773, $\mathrm{P}=0.027$ ).

Besides, in Fuhrman nuclear grade 1-2 group, tumor size retained its prognostic value and was statistically linked to all OS, CSS, and RFS. NLR and the presence of symptoms were respectively independently associated with CSS and RFS. Details are shown in Table 5.

\section{Analysis of MetS components}

We further analyzed the correlation between MetS components and CSS. Table 6 showed univariate and 
Table 1 Comparison of baseline clinicopathological data of patients with localized ccRCC by MetS

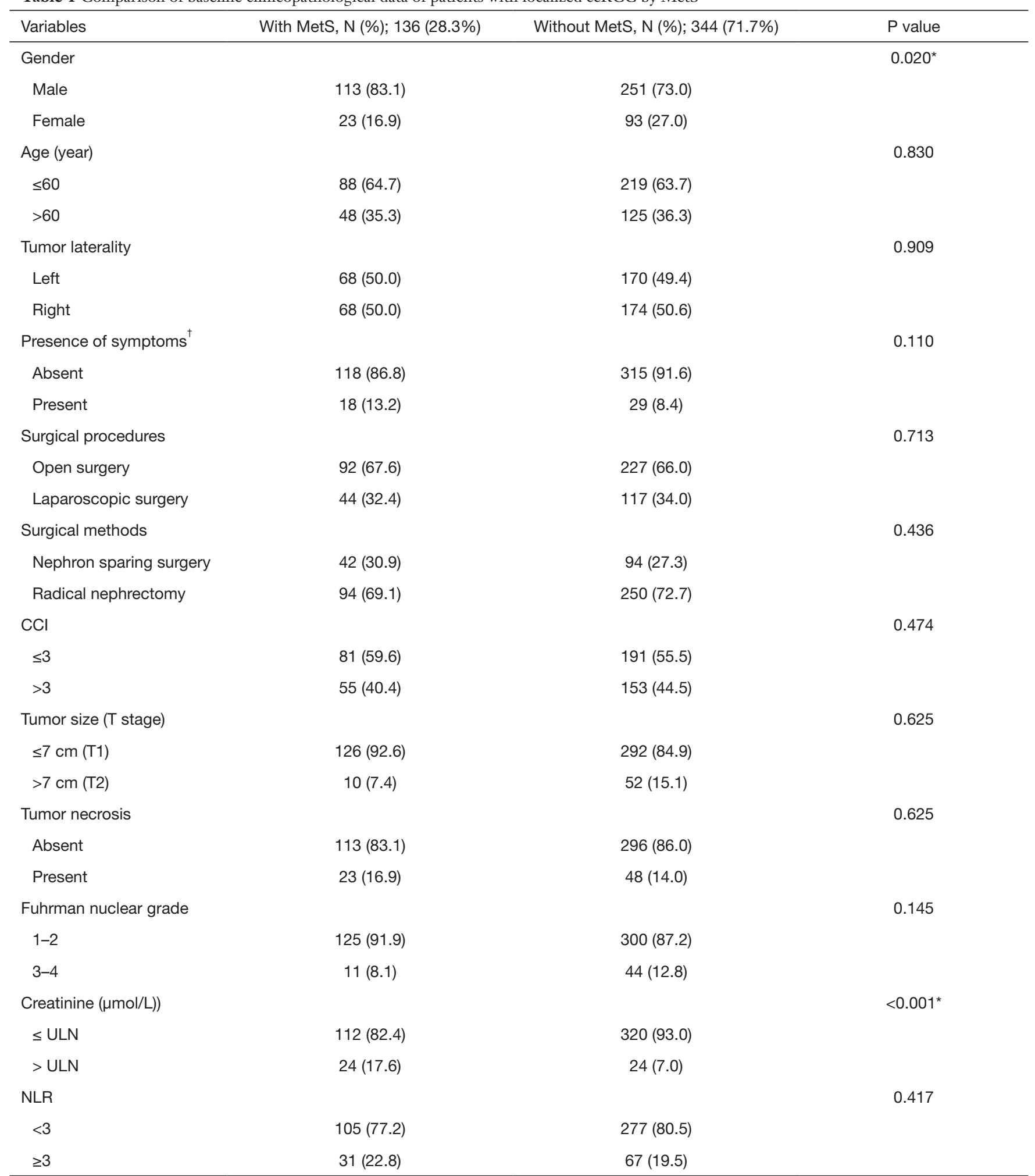

${ }^{\dagger}$ Presence of symptoms: one or more of the following symptoms including flank pain, hematuria, fever and weight loss were considered with positive symptoms in this study; ${ }^{*} \mathrm{P}<0.05$. $\mathrm{CCl}$, Charlson comorbidity index; ULN, upper limit of normal; NLR, neutrophil-lymphocyte ratio; MetS, metabolic syndrome. 

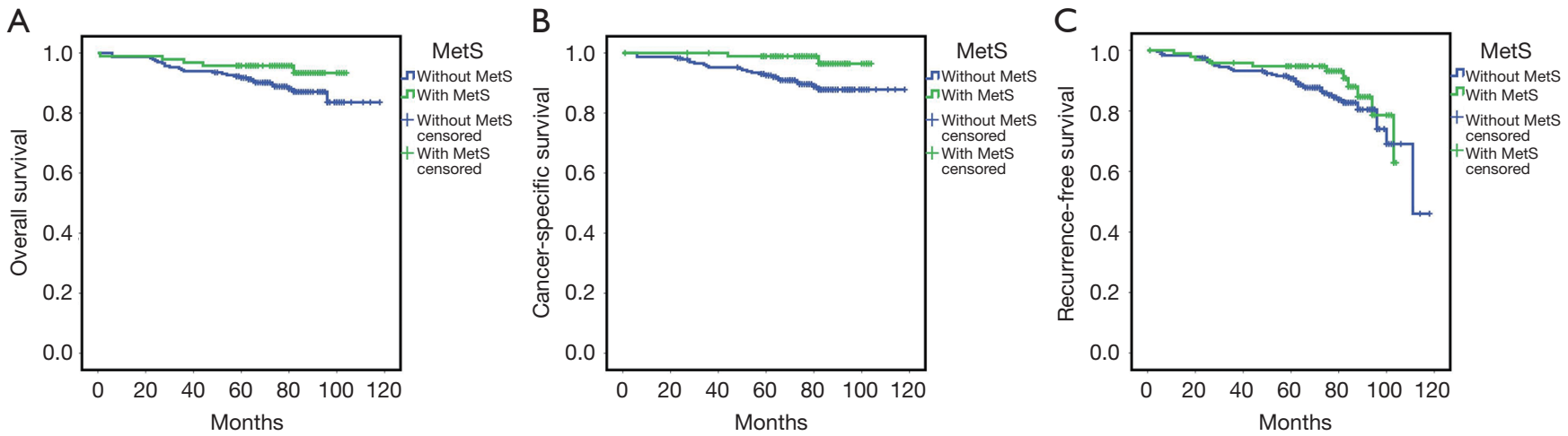

Figure $1 \mathrm{OS}$, CSS and RFS curves for localized ccRCC patients with and without MetS. Localized ccRCC patients with MetS had higher CSS $(\mathrm{P}=0.014$, B) than patients without MetS. While it was not statistically significant in OS $(\mathrm{P}=0.084, \mathrm{~A})$ and $\mathrm{RFS}(\mathrm{P}=0.214, \mathrm{C})$. Curves were drawn by SPSS software using Kaplan-Meier survival analysis. CSS, cancer-specific survival; MetS, metabolic syndrome; OS, overall survival; RFS, recurrence-free survival.

Table 2 Univariate analysis of factors associated with prognosis (OS, CSS and RFS) in patients with localized ccRCC

\begin{tabular}{|c|c|c|c|c|c|c|}
\hline Variables & \multicolumn{2}{|l|}{ OS } & \multicolumn{2}{|l|}{ CSS } & \multicolumn{2}{|l|}{ RFS } \\
\hline Gender (female/male) & $0.741(0.320-1.713)$ & 0.483 & $0.964(0.405-2.294)$ & 0.934 & $0.796(0.417-1.519)$ & 0.488 \\
\hline Age $(>60 / \leq 60)$ & $3.388(1.633-7.027)$ & $0.001^{*}$ & $2.875(1.305-6.337)$ & $0.009^{*}$ & $1.490(0.857-2.588)$ & 0.157 \\
\hline Tumor laterality (left/right) & $1.829(0.894-3.742)$ & 0.098 & $1.761(0.799-3.880)$ & 0.160 & $1.009(0.585-1.740)$ & 0.975 \\
\hline Symptoms $^{\dagger}$ (yes/no) & $2.015(0.775-5.242)$ & 0.151 & $2.691(1.014-7.144)$ & $0.047^{*}$ & $2.869(1.467-5.610)$ & $0.002^{*}$ \\
\hline Tumor necrosis (yes/no) & $1.385(0.569-3.370)$ & 0.473 & $0.780(0.234-2.602)$ & 0.687 & $1.303(0.632-2.686)$ & 0.473 \\
\hline Creatinine $(\leq$ ULN/> ULN) & $1.045(0.318-3.434)$ & 0.942 & $0.859(0.203-3.634)$ & 0.836 & $0.799(0.287-2.221)$ & 0.667 \\
\hline $\begin{array}{l}\text { Surgery } \\
\text { (open/ laparoscopic) }\end{array}$ & $0.608(0.273-1.355)$ & 0.224 & $0.675(0.283-1.609)$ & 0.375 & $0.658(0.350-1.235)$ & 0.192 \\
\hline $\mathrm{CCl}(>3 / \leq 3)$ & $2.922(1.383-6.170)$ & $0.005^{\star}$ & $2.530(1.128-5.677)$ & $0.024^{*}$ & $1.410(0.813-2.444)$ & 0.221 \\
\hline Fuhrman grade $(3-4 / \leq 1-2)$ & $1.936(1.111-3.373)$ & $0.020^{\star}$ & $1.631(0.879-3.027)$ & 0.121 & $2.211(1.441-3.392)$ & $<0.001^{\star}$ \\
\hline $\operatorname{NLR}(\geq 3 /<3)$ & $2.727(1.346-5.524)$ & $0.005^{\star}$ & $3.462(1.601-7.485)$ & $0.002^{*}$ & $1.975(1.113-3.505)$ & $0.020^{*}$ \\
\hline MetS (yes/no) & $0.441(0.170-1.146)$ & 0.093 & $0.198(0.047-0.839)$ & $0.028^{*}$ & $0.657(0.337-1.283)$ & 0.219 \\
\hline
\end{tabular}

${ }^{\dagger}$ Presence of symptoms: one or more of the following symptoms including flank pain, hematuria, fever and weight loss were considered with positive symptoms in this study; ${ }^{*} \mathrm{P}<0.05$. $\mathrm{CCI}$, Charlson comorbidity index; CSS, cancer-specific survival; ULN, upper limit of normal; NLR, neutrophil-lymphocyte ratio; MetS, metabolic syndrome; OS, overall survival; RFS, recurrence-free survival. 
Table 3 Multivariate analysis of factors affecting prognosis (OS, CSS and RFS) in patients with localized ccRCC

\begin{tabular}{|c|c|c|c|c|c|c|}
\hline Variables & \multicolumn{2}{|l|}{ OS } & \multicolumn{2}{|l|}{ CSS } & \multicolumn{2}{|l|}{ RFS } \\
\hline Age $(>60 / \leq 60)$ & $3.413(0.455-25.616)$ & 0.233 & $2.641(0.343-20.313)$ & 0.351 & - & - \\
\hline Tumor size $(>7 / \leq 7 \mathrm{~cm})$ & $2.559(0.997-6.573)$ & 0.051 & 4.166 (1.632-10.632) & $0.003^{*}$ & 3.702 (1.905-7.194) & $<0.001^{*}$ \\
\hline Symptoms $^{\dagger}$ (yes/no) & $1.997(0.734-5.432)$ & 0.176 & $2.841(1.040-7.762)$ & $0.042^{*}$ & $2.485(1.246-4.956)$ & $0.010^{*}$ \\
\hline Fuhrman grade (3-4/1-2) & $1.429(0.574-3.556)$ & 0.443 & - & - & $1.586(0.807-3.118)$ & 0.181 \\
\hline $\begin{array}{l}\text { Surgery } \\
\text { (nephron sparing/radical) }\end{array}$ & - & - & - & - & $1.471(0.661-3.274)$ & 0.344 \\
\hline $\operatorname{NLR}(\geq 3 /<3)$ & $1.967(0.947-4.085)$ & 0.070 & $3.096(1.394-6.876)$ & $0.005^{\star}$ & $1.509(0.833-2.734)$ & 0.175 \\
\hline
\end{tabular}

${ }^{\dagger}$ Presence of symptoms: one or more of the following symptoms including flank pain, hematuria, fever and weight loss were considered with positive symptoms in this study; ${ }^{*} \mathrm{P}<0.05$. $\mathrm{CCl}$, Charlson comorbidity index; CSS, cancer-specific survival; NLR, neutrophillymphocyte ratio; MetS, metabolic syndrome; OS, overall survival; RFS, recurrence-free survival; $\mathrm{HR}$, hazard ratio; $\mathrm{Cl}$, confidence interval.

Table 4 Univariate analysis of subgroups with different Fuhrman grades

\begin{tabular}{|c|c|c|c|c|c|c|}
\hline Variables & \multicolumn{3}{|c|}{ Fuhrman grade [1-2], $P$ value } & \multicolumn{3}{|c|}{ Fuhrman grade [3-4], $P$ value } \\
\hline Gender (female/male) & 0.781 & 0.927 & 0.877 & 0.430 & 0.747 & 0.206 \\
\hline Age $(>60 / \leq 60)$ & $0.004^{*}$ & $0.024^{*}$ & 0.790 & 0.470 & 0.442 & 0.252 \\
\hline Tumor laterality (left/right) & 0.284 & 0.370 & 0.550 & 0.176 & 0.196 & 0.392 \\
\hline Symptoms $^{\dagger}$ (yes/no) & 0.108 & 0.055 & $0.005^{\star}$ & 0.875 & 0.747 & 0.458 \\
\hline Tumor necrosis (yes/no) & 0.676 & 0.354 & 0.392 & 0.738 & 0.91 & 0.833 \\
\hline Creatinine ( $\leq$ ULN/> ULN) & 0.831 & 0.974 & 0.608 & 0.26 & 0.761 & 0.533 \\
\hline Surgery (open/laparoscopic) & 0.243 & 0.444 & 0.443 & 0.924 & 0.812 & 0.423 \\
\hline $\operatorname{NLR}(\geq 3 /<3)$ & $0.019^{*}$ & $0.005^{\star}$ & 0.055 & 0.459 & 0.309 & 0.514 \\
\hline MetS (yes/no) & 0.059 & $0.031^{*}$ & 0.168 & 0.383 & 0.713 & 0.406 \\
\hline
\end{tabular}

${ }^{\dagger}$ Presence of symptoms: one or more of the following symptoms including flank pain, hematuria, fever and weight loss were considered with positive symptoms in this study; ${ }^{*} \mathrm{P}<0.05$. CCI, Charlson comorbidity index; CSS, cancer-specific survival; ULN, upper limit of normal; NLR, neutrophil-lymphocyte ratio; MetS, metabolic syndrome; OS, overall survival; RFS, recurrence-free survival.

multivariate analysis of MetS components. No difference was found between CSS and hypertension, diabetes mellitus as well as dyslipidemia (also showed in Figure 3). Figure $4 A$ shows patients with a higher BMI $(\mathrm{BMI} \geq 25)$ may had better CSS $(\mathrm{P}=0.044)$. As a MetS component,
BMI was calculated in multivariate analysis with other factors in Table 3 (age, tumor size, symptom, CCI, NLR were statistically significant in univariate analysis). Table 6 shows although $\mathrm{BMI} \geq 25$ may be related to better CSS, it was not an independent factor for CSS $(\mathrm{P}=0.189)$. 
A

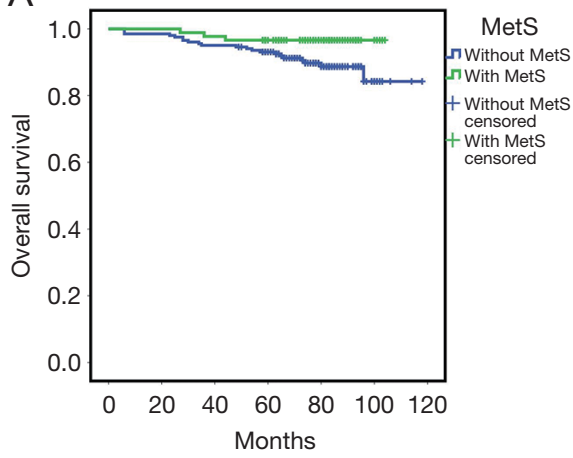

B

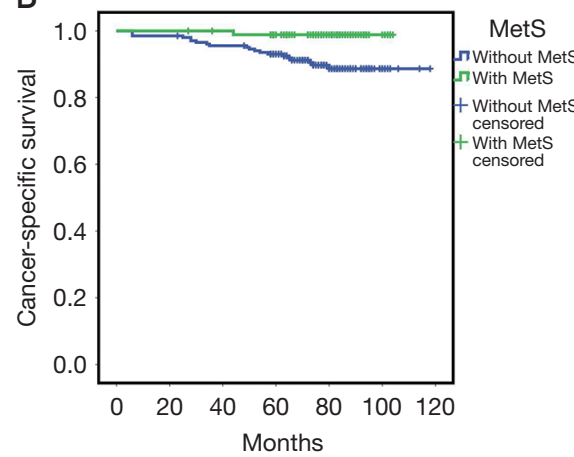

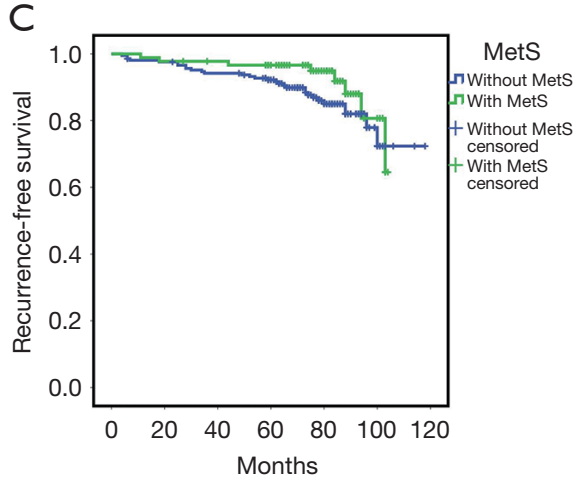

Figure 2 OS, CSS and RFS curves for Fuhrman nuclear grade 1-2 localized ccRCC patients with and without MetS. Fuhrman nuclear grade 1-2 localized ccRCC patients with MetS had higher OS $(\mathrm{P}=0.046, \mathrm{~A})$ and CSS $(\mathrm{P}=0.009, \mathrm{~B})$ than patients without MetS. While it was not statistically significant in RFS $(\mathrm{P}=0.162, \mathrm{C})$. Curves were drawn by SPSS software using Kaplan-Meier survival analysis. CSS, cancerspecific survival; MetS, metabolic syndrome; OS, overall survival; RFS, recurrence-free survival.

Table 5 Multivariate analysis of subjects of Fuhrman grades 1-2

\begin{tabular}{|c|c|c|c|c|c|c|}
\hline Variables & \multicolumn{2}{|l|}{ OS } & \multicolumn{2}{|l|}{ CSS } & \multicolumn{2}{|l|}{ RFS } \\
\hline Age $(>60 / \leq 60)$ & 3.782 (0.779-18.359) & 0.099 & $2.099(0.856-5.146)$ & 0.105 & - & - \\
\hline Tumor size $(>7 / \leq 7)$ & 3.909 (1.321-11.570) & $0.014^{\star}$ & $6.174(2.252-16.926)$ & $<0.001^{\star}$ & $6.957(3.426-14.127)$ & $<0.001^{\star}$ \\
\hline Symptoms $^{\dagger}$ (yes/no) & - & - & - & - & $3.132(1.419-6.913)$ & $0.005^{\star}$ \\
\hline $\operatorname{NLR}(\geq 3 /<3)$ & $1.982(0.853-4.603)$ & 0.112 & 2.682 (1.096-6.562) & $0.031^{*}$ & - & - \\
\hline MetS (yes/no) & - & - & $0.103(0.014-0.773)$ & $0.027^{*}$ & - & - \\
\hline
\end{tabular}

${ }^{\dagger}$ Presence of symptoms: one or more of the following symptoms including flank pain, hematuria, fever and weight loss were considered with positive symptoms in this study; ${ }^{*} \mathrm{P}<0.05$. $\mathrm{CCl}$, Charlson comorbidity index; CSS, cancer-specific survival; NLR, neutrophillymphocyte ratio; MetS, metabolic syndrome; OS, overall survival; RFS, recurrence-free survival; HR, hazard ratio; $\mathrm{Cl}$, confidence interval.

Table 6 Univariate and multivariate analysis of MetS components

\begin{tabular}{|c|c|c|c|c|}
\hline Variables & \multicolumn{2}{|c|}{ Univariate analysis of CSS } & \multicolumn{2}{|c|}{ Multivariate analysis of CSS } \\
\hline MetS (yes/no) & $0.198(0.047-0.839)$ & $0.028^{*}$ & $0.168(0.039-0.728)$ & $0.017^{*}$ \\
\hline Hypertension (no/yes) & $0.629(0.264-1.499)$ & 0.296 & - & - \\
\hline Diabetes mellitus (no/yes) & $0.976(0.336-2.832)$ & 0.964 & - & - \\
\hline Dyslipidemia (no/yes) & $0.704(0.325-1.522)$ & 0.372 & - & - \\
\hline
\end{tabular}

The results of multivariate analysis were calculated independently of metabolic syndrome component (BMI) and in combination with other factors in Table 3 (age, tumor size, symptom, CCI, NLR). ${ }^{*} \mathrm{P}<0.05$. BMI, body mass index; CCl, Charlson comorbidity index; CSS, cancerspecific survival; NLR, neutrophil-lymphocyte ratio; MetS, metabolic syndrome; HR, hazard ratio; Cl, confidence interval. 

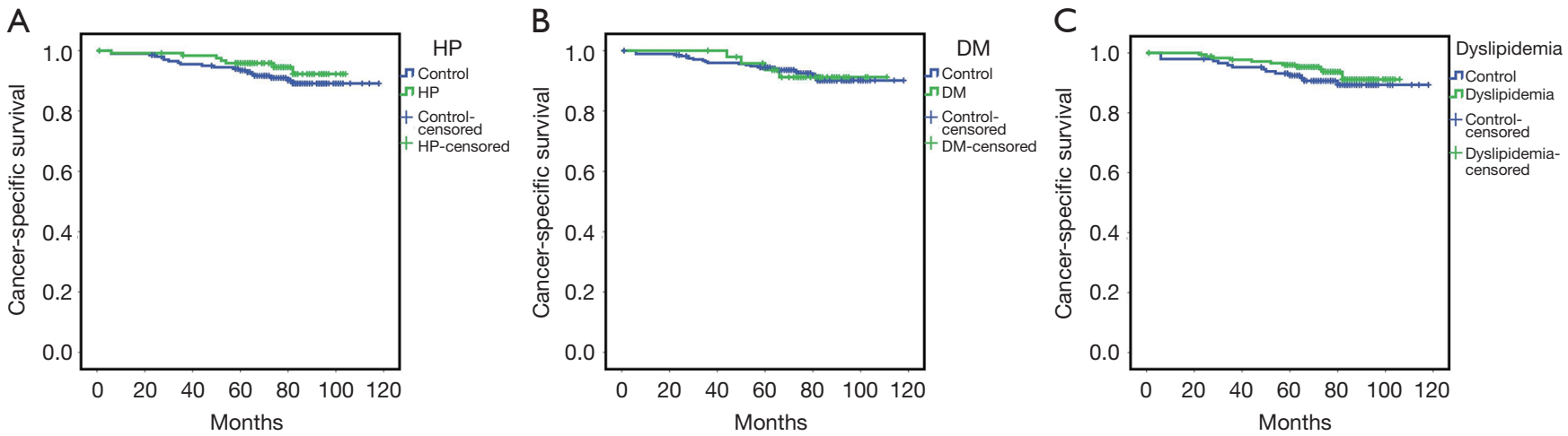

Figure 3 CSS curves for localized ccRCC patients with MetS components (hypertension, diabetes mellitus and dyslipidemia). In this image, we studied whether localized ccRCC patients with diseases as components of MetS (HP, DM and dyslipidemia) will affect CSS or not. However, we could not find the association between CSS and hypertension ( $\mathrm{P}=0.291$, A), diabetes mellitus ( $\mathrm{P}=0.964$, B) or dyslipidemia $(\mathrm{P}=0.369, \mathrm{C})$. Curves were drawn by SPSS software using Kaplan-Meier survival analysis. CSS, cancer-specific survival; DM, diabetes mellitus; HP, hypertension; MetS, metabolic syndrome.
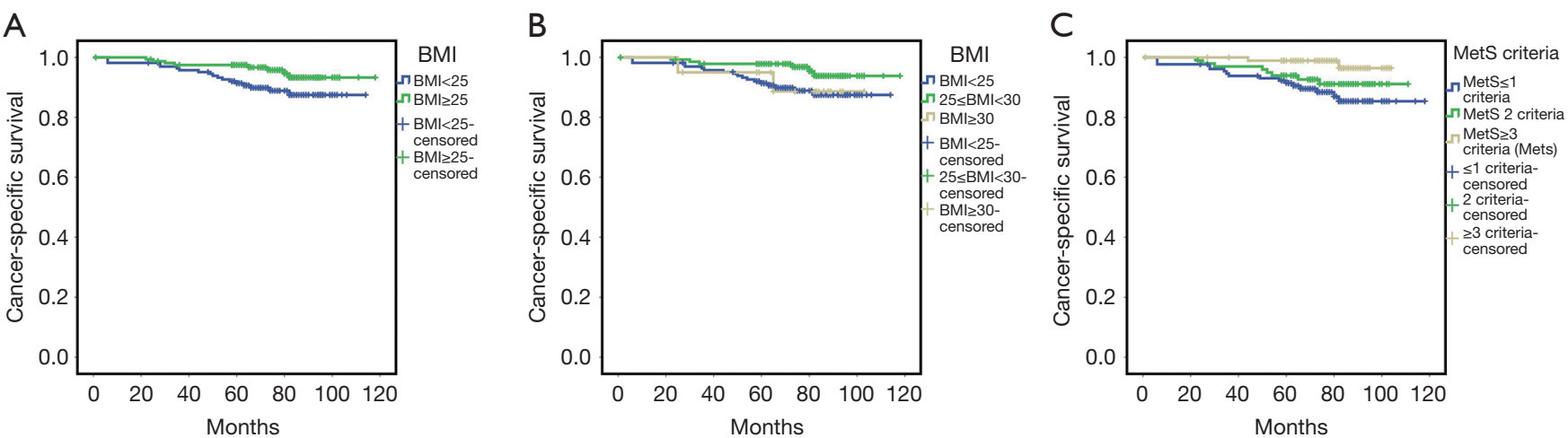

Figure 4 CSS curves for localized ccRCC patients with MetS components (BMI) and numbers of MetS criteria. In (A,B), we studied whether localized ccRCC patients with disease as components of MetS (overweight/obesity) will affect CSS or not. The result showed patients with a higher $\mathrm{BMI}(\mathrm{BMI} \geq 25)$ may had better CSS $(\mathrm{P}=0.044, \mathrm{~A})$. Then, we divided $\mathrm{BMI}$ into three groups $(\mathrm{BMI}<25,25 \leq \mathrm{BMI}<30$, $\mathrm{BMI} \geq 30$ ) and found patients with $25 \leq \mathrm{BMI}<30$ had best $\mathrm{CSS}$ ( $\mathrm{B} ; 25 \leq \mathrm{BMI}<30$ vs. $\mathrm{BMI}<25: \mathrm{P}=0.029 ; 25 \leq \mathrm{BMI}<30$ vs. $\mathrm{BMI} \geq 30$ : $\mathrm{P}=0.313$ ). In $(\mathrm{C})$, it showed patients with Mets (met $\geq 3$ of the 4 MetS criteria) had higher CSS than those who only met 2 or $<2$ criteria $(\mathrm{P}=0.022$ ). Curves were drawn by SPSS software using Kaplan-Meier survival analysis. BMI, body mass index; CSS, cancer-specific survival; MetS, metabolic syndrome.

In Figure $4 B$, we divided BMI into three groups (BMI $<25,25 \leq$ BMI $<30$, BMI $\geq 30$ ) and utilized Kaplan-Meier curves to analyze the relationship between each group and CSS. The group $25 \leq \mathrm{BMI}<30$ showed the best CSS $(\mathrm{P}=0.029)$.

Since the diagnosis of MetS requires 3 or 4 criteria to be met, will there be any change in CSS if patients only meet $0-1$ criterion, or only meet 2 criteria? We used Kaplan-Meier curves to analyze the relationship and showed results in Figure 4C. Patients who met 3-4 of the 4 criteria for MetS had higher CSS than those who met only 2 or $<2$ criteria.

\section{Discussion}

MetS has recently been linked to several cancers, including breast cancer (14), liver cancer (14), colorectal cancer (15), bladder cancer (16), prostate cancer (17), endometrial 
cancer (14) and RCC (18).

\section{Characters of MetS cases}

In the present study, we found more males had MetS than females. There was a major sex disparity in the epidemiology of MetS and this disparity was influenced by geographic variation. Some studies conducted in China indicated a higher prevalence of MetS in women, both rural and urban, than men $(19,20)$. On the contrary, the United States and Japan both reported a prevalence of MetS in men $(21,22)$. Our result may be due to gender differences in the morbidity of RCC, with a male-to-female ratio of 2.8:1.

MetS was previously found related to the differentiation of tumor cells. In colon (23) and prostate cancer $(10,24)$, MetS and its components were associated with a more aggressive tumor type, although results were controversial for breast cancer $(25,26)$. However, Wei et al. found MetS related to better differentiation of gastric cancer cells (7). A study of 310 Turkish patients with RCC revealed that concomitant MetS status was significantly connected with higher nuclear grade and larger tumor size (27), which differed from our findings of no difference in tumor grade or size by MetS status.

\section{Components of MetS}

Whether the components of MetS have an independent effect on the prognosis of renal cancer is still controversial. Eskelinen $e t a l$. analyzed risk associations between MetS components and prognosis of 13,873 Finnish RCC cases, and found hypertension was independently associated with worse RCC survival (28). Type 2 diabetes mellitus among women (29) and high BMI and blood pressure among men (30) have been found as independent risk factors for RCC. In a study of 970 ccRCC cases, Parker et al. discovered that overweight and obese people were less likely to have tumors with classic aggressive features than those with normal BMI (31). However, Kriegmair et al. discovered none of the MetS components were independent prognostic factors for RCC (32).

In our study, no difference was found between CSS and hypertension, diabetes mellitus or dyslipidemia. Although BMI $\geq 25$ may be related to favorable CSS, it was not an independent factor for CSS. Group $25 \leq \mathrm{BMI}<30$ may contribute more to the favorable CSS of MetS. However, patients who met 3-4 of the 4 criteria for MetS had higher CSS than those who met only 2 or $<2$ criteria. In other words, our conclusions support that MetS is an independent favorable prognostic factor for CSS in ccRCC.

\section{MetS and cancer progress}

Numerous mechanisms have been proposed to explain the role of MetS in cancers, including insulin resistance, inflammation, angiogenesis, cell-stroma interaction, and many other important aspects (33). The insulin-like growth factor (IGF) system, composed of IGF-1, IGF-2, and their receptors, IGF-1R, IGF-2R, as well as IGF binding proteins (IGFBPs), is implicated in the regulation of the malignant phenotype by its effects on proliferation, differentiation, and apoptosis (34). Insulin resistance is consistently accompanied by hyperinsulinemia, which enhances the activity of IGF by inhibiting the synthesis of IGFBPs. The response of IGF-1R or insulin receptor substrate-1 (IRS-1) to IGF-1 results in the activation of phosphatidylinositol-3 kinase $(\mathrm{PI} 3 \mathrm{~K})$ and mitogen-activated protein kinase signaling pathways. IGF-1 plays a part in the promotion of mitosis, cell proliferation and migration and inhibition of apoptosis (34). Also, despite the diverse metabolic effects, insulin is a growth factor causing pathological cell proliferation.

In addition, MetS is closely linked to obesity. Hyperplasia and hypertrophy of adipocytes in obesity are common and lead to tissue hypoxia and the induction of a series of pro-inflammatory cytokines, such as tumor necrosis factor- $\alpha$ (TNF- $\alpha$ ), interleukin-6 (IL-6), IL-8 and IL-10 (33). MetS patients with high levels of certain pro-inflammatory cytokines may be susceptible to the development of tumors. Adiponectin, secreted by white adipose tissues, could inhibit tumor growth via activating AMP-activated protein kinase (AMPK) (AMP: adenosine monophosphate), thereby downregulating the mammalian target of rapamycin pathways (35). Hopxia and TNF- $\alpha$ can down regulate the activity of adiponectin gene promoter (36); adiponectin levels could be decreased in MetS patients, thereby increasing the risk of RCC. Furthermore, serum adiponectin levels were discovered to reduce in RCC (37); adiponectin levels and tumor sizes were strongly correlated with RCC metastasis (37).

\section{MetS and cancer prognosis}

The impact of MetS on the survival of cancers is complicated. Some studies have illustrated that MetS is negatively related to other cancers' outcomes. Hu et al. 
discovered via a study of 3,012 gastric cancer patients that the median survival time (MST) was significantly shorter for MetS patients than MetS-free patients and the coexistence of MetS before surgery was associated with a 2.3 -fold increased risk for gastric cancer mortality (38). Kriegmair et al. discovered MetS negatively impacted the RFS of RCC (32). However, this study covered various subtypes of RCC and included cases of lymph node metastasis, which might be the reason for the inconsistency with the conclusions of present study.

In contrast, Wei et al. found that older patients or those with proximal tumors benefitted from MetS in early-stage gastric cancer and found a correlation between MetS and better differentiation of gastric cancer cells (7). Zou et al. also found MetS associated with better prognosis in patients with tongue squamous cell carcinoma (8). Wen and colleagues also discovered MetS was a significant and independent predictor for better survival in patients with resectable esophageal squamous cell carcinoma. The 3 -year OS and 5-year OS for patients with and without MetS were $75.0 \%$ versus $57.8 \%$ and $65.1 \%$ versus $44.6 \%$, respectively (39).

We also found MetS was associated with better CSS in patients with localized ccRCC and the result was similar in Fuhrman nuclear grade 1-2 ccRCC patients. BMI $\geq 25$ may be related to favorable CSS in univariate analysis, and group $25 \leq \mathrm{BMI}<30$ may contribute more to the favorable CSS of MetS. Better outcomes of ccRCC patients with MetS in our study might be a result of a favorable role played by obesity, which is a vital constituent of MetS. Patients with higher BMI might have better nutritional status and potential survival advantage (40). Especially for organconfined RCC, being overweight improves CSS (41). In addition, the impact of MetS on cells, tissues, organs or even the whole body is chronic and continuous, which requires time to ascertain the influence. The properties of tumor cells may directly determine the outcome of patients with advanced ccRCC, despite the effects of MetS. For patients with localized ccRCC, their prolonged survival may give MetS an opportunity to play its role, thereby altering the final outcome. Finally, Morgan et al. found nutritional deficiency was associated with increased mortality in patients undergoing surgery for localized RCC (42). MetS patients may seem more resistant to chronic consumption from cancers.

\section{Selection of diagnostic criteria for MetS and limitation of study}

The definitions of MetS from the International Diabetes
Federation (IDF) and revised National Cholesterol Education Program-Adult Treatment Panel (ATP III*) are commonly used. However, the agreement in the diagnosis of MetS was $75.7 \%$ with the CDS and ATP III* definitions and $73.7 \%$ with the CDS and IDF criteria, both lower than the agreement of $88.6 \%$ with the IDF and ATP III* criteria (43). MetS was more easily diagnosed with the IDF or ATP III* criteria than the CDS criteria (43). So, using different definitions may result in inconsistency in the prognostic value of MetS. The CDS criteria were thought to be the compound product after integrating consideration of metabolic abnormalities and Chinese characteristics. In addition, we could not get records for abdomen circumference in clinical data. Founded on the comprehensive consideration above, we deemed the CDS definition appropriate.

One limitation of our study is its retrospective nature from a single center. Therefore, in order to generalize our conclusions to other communities, studies from other cohorts should be carried out. In addition, we did not investigate the different forms of MetS, which may have an influence on the results. Additional studies are needed to investigate the impact of specific forms of MetS on survival with localized RCC to better clarify this issue.

\section{Conclusions}

In this retrospective study, we intended to evaluate the prognostic value of MetS in patients with localized ccRCC. We found MetS to be an independent favorable prognostic factor of CSS in patients with localized RCC.

\section{Acknowledgments}

None.

\section{Footnote}

Conflicts of Interest: The authors have no conflicts of interest to declare.

Ethical Statement: The authors are accountable for all aspects of the work in ensuring that questions related to the accuracy or integrity of any part of the work are appropriately investigated and resolved. This study was approved by the institutional ethics committee of Peking University First Hospital \{approval number: 2016[1037]\}. Because it was a retrospective analysis of routine data, a 
waiver of wrote informed consent was granted from the ethics committee. Patient records or information were anonymous and de-identified prior to analysis.

\section{References}

1. Capitanio U, Bensalah K, Bex A, et al. Epidemiology of Renal Cell Carcinoma. Eur Urol 2019;75:74-84.

2. Protzel C, Maruschke M, Hakenberg OW. Epidemiology, Aetiology, and Pathogenesis of Renal Cell Carcinoma. European Urology Supplements 2012;11:52-9.

3. Moch H, Cubilla AL, Humphrey PA, et al. The 2016 WHO Classification of Tumours of the Urinary System and Male Genital Organs-Part A: Renal, Penile, and Testicular Tumours. Eur Urol 2016;70:93-105.

4. Lam JS, Leppert JT, Figlin RA, et al. Surveillance following radical or partial nephrectomy for renal cell carcinoma. Curr Urol Rep 2005;6:7-18.

5. Reaven GM. Banting lecture 1988. Role of insulin resistance in human disease. Diabetes 1988;37:1595-607.

6. Thomas GN, Ho SY, Janus ED, et al. The US National Cholesterol Education Programme Adult Treatment Panel III (NCEP ATP III) prevalence of the metabolic syndrome in a Chinese population. Diabetes Res Clin Pract 2005;67:251-7.

7. Wei XL, Qiu MZ, Lin HX, et al. Patients with old age or proximal tumors benefit from metabolic syndrome in early stage gastric cancer. PLoS One 2014;9:e89965.

8. Zou L, Liu TR, Yang AK. Metabolic syndrome is associated with better prognosis in patients with tongue squamous cell carcinoma. Chin J Cancer 2015;34:184-8.

9. Reeves KW, McLaughlin V, Fredman L, et al. Components of metabolic syndrome and risk of breast cancer by prognostic features in the study of osteoporotic fractures cohort. Cancer Causes Control 2012;23:1241-51.

10. Xiang YZ, Xiong H, Cui ZL, et al. The association between metabolic syndrome and the risk of prostate cancer, high-grade prostate cancer, advanced prostate cancer, prostate cancer-specific mortality and biochemical recurrence. J Exp Clin Cancer Res 2013;32:9.

11. Bulut S, Aktas BK, Erkmen AE, et al. Metabolic syndrome prevalence in renal cell cancer patients. Asian Pac J Cancer Prev 2014;15:7925-8.

12. Group Cmadsmss. Chinese medical association diabetes society recommendations for metabolic syndrome. Chin J Diabetes 2004:5-10.

13. Campbell MF, Walsh PC, Wein AJ, et al. CampbellWalsh Urology. 11 ed., vol 2. Saunders Elsevier, 2015.
14. Esposito K, Chiodini P, Colao A, et al. Metabolic syndrome and risk of cancer: a systematic review and metaanalysis. Diabetes Care 2012;35:2402-11.

15. Ulaganathan V, Kandiah M, Zalilah MS, et al. Colorectal cancer and its association with the metabolic syndrome: a Malaysian multi-centric case-control study. Asian Pac J Cancer Prev 2012;13:3873-7.

16. Häggström C, Stocks T, Rapp K, et al. Metabolic syndrome and risk of bladder cancer: prospective cohort study in the metabolic syndrome and cancer project (MeCan). Int J Cancer 2011;128:1890-8.

17. Zhang JQ, Geng H, Ma M, et al. Metabolic Syndrome Components are Associated with Increased Prostate Cancer Risk. Med Sci Monit 2015;21:2387-96.

18. Häggström C, Rapp K, Stocks T, et al. Metabolic factors associated with risk of renal cell carcinoma. PLoS One 2013;8:e57475.

19. Yu S, Guo X, Yang H, et al. An update on the prevalence of metabolic syndrome and its associated factors in rural northeast China. BMC Public Health 2014;14:877.

20. Xi B, He D, Hu Y, et al. Prevalence of metabolic syndrome and its influencing factors among the Chinese adults: the China Health and Nutrition Survey in 2009. Prev Med 2013;57:867-71.

21. Ishii S, Tanaka T, Akishita $M$, et al. Metabolic syndrome, sarcopenia and role of sex and age: cross-sectional analysis of Kashiwa cohort study. PLoS One 2014;9:e112718.

22. Mabry RM, Reeves MM, Eakin EG, et al. Gender differences in prevalence of the metabolic syndrome in Gulf Cooperation Council Countries: a systematic review. Diabet Med 2010;27:593-7.

23. Healy LA, Howard JM, Ryan AM, et al. Metabolic syndrome and leptin are associated with adverse pathological features in male colorectal cancer patients. Colorectal Dis 2012;14:157-65.

24. Caliskan S, Kaba S, Ozsoy E, et al. The effect of metabolic syndrome on prostate cancer final pathology. J Cancer Res Ther 2019;15:S47-50.

25. Healy LA, Ryan AM, Carroll P, et al. Metabolic syndrome, central obesity and insulin resistance are associated with adverse pathological features in postmenopausal breast cancer. Clin Oncol (R Coll Radiol) 2010;22:281-8.

26. Colonna SV, Douglas Case L, Lawrence JA. A retrospective review of the metabolic syndrome in women diagnosed with breast cancer and correlation with estrogen receptor. Breast Cancer Res Treat 2012;131:325-31.

27. Ozbek E, Otunctemur A, Sahin S, et al. Renal cell carcinoma is more aggressive in Turkish patients with 
the metabolic syndrome. Asian Pac J Cancer Prev 2013;14:7351-4.

28. Eskelinen TJ, Kotsar A, Tammela TLJ, et al. Components of metabolic syndrome and prognosis of renal cell cancer. Scand J Urol 2017;51:435-41.

29. Joh HK, Willett WC, Cho E. Type 2 diabetes and the risk of renal cell cancer in women. Diabetes Care 2011;34:1552-6.

30. Chow WH, Gridley G, Fraumeni JF Jr, et al. Obesity, hypertension, and the risk of kidney cancer in men. $\mathrm{N}$ Engl J Med 2000;343:1305-11.

31. Parker AS, Lohse CM, Cheville JC, et al. Greater body mass index is associated with better pathologic features and improved outcome among patients treated surgically for clear cell renal cell carcinoma. Urology 2006;68:741-6.

32. Kriegmair MC, Mandel P, Porubsky S, et al. Metabolic Syndrome Negatively Impacts the Outcome of Localized Renal Cell Carcinoma. Horm Cancer 2017;8:127-34.

33. Zhang GM, Zhu Y, Ye DW. Metabolic syndrome and renal cell carcinoma. World J Surg Oncol 2014;12:236.

34. Ibrahim YH, Yee D. Insulin-like growth factor-I and cancer risk. Growth Horm IGF Res 2004;14:261-9.

35. Sugiyama M, Takahashi H, Hosono K, et al. Adiponectin inhibits colorectal cancer cell growth through the AMPK/ mTOR pathway. Int J Oncol 2009;34:339-44.

36. Ukkola O, Santaniemi M. Adiponectin: a link between excess adiposity and associated comorbidities? J Mol Med (Berl) 2002;80:696-702.

Cite this article as: Liu Z, Wang H, Zhang L, Li S, Fan Y, Meng Y, Hu S, Zhang Q, He Z, Zhou L, Han W, Yu W, Jin J. Metabolic syndrome is associated with improved cancer-specific survival in patients with localized clear cell renal cell carcinoma. Transl Androl Urol 2019;8(5):507-518. doi: 10.21037/ tau.2019.10.04
37. Pinthus JH, Kleinmann N, Tisdale B, et al. Lower plasma adiponectin levels are associated with larger tumor size and metastasis in clear-cell carcinoma of the kidney. Eur Urol 2008;54:866-73.

38. Hu D, Peng F, Lin X, et al. Preoperative Metabolic Syndrome Is Predictive of Significant Gastric Cancer Mortality after Gastrectomy: The Fujian Prospective Investigation of Cancer (FIESTA) Study. EBioMedicine 2017;15:73-80.

39. Wen YS, Huang C, Zhang X, et al. Impact of metabolic syndrome on the survival of Chinese patients with resectable esophageal squamous cell carcinoma. Dis Esophagus 2016;29:607-13.

40. Choi Y, Park B, Jeong BC, et al. Body mass index and survival in patients with renal cell carcinoma: a clinical-based cohort and meta-analysis. Int J Cancer 2013;132:625-34.

41. Waalkes S, Merseburger AS, Kramer MW, et al. Obesity is associated with improved survival in patients with organconfined clear-cell kidney cancer. Cancer Causes Control 2010;21:1905-10.

42. Morgan TM, Tang D, Stratton KL, et al. Preoperative nutritional status is an important predictor of survival in patients undergoing surgery for renal cell carcinoma. Eur Urol 2011;59:923-8.

43. Gao X WX, Tang Z, Gao FK. Application of three criteria for metabolic syndrome among the elderly in urban community of Beijing. Chin J Geriatr 2012;31:248-50. 